\title{
SURGICALLY CORRECTABLE ADRENAL-DEPENDENT HYPER- TENSION: A REPORT OF FIVE CASES
}

\author{
*F. OFEI, R. DARKO ${ }^{1}$, J. APPIAH-KUSI, H. ADUFUL ${ }^{1}$, H. BADDOO ${ }^{2}$, Y. ADU-

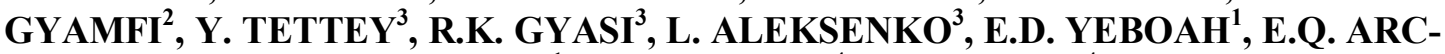 \\ HAMPONG ${ }^{1}$, A.A. YEBOAH ${ }^{4}$ and E. OGOE ${ }^{4}$ \\ Departments of Medicine \& Therapeutics, ${ }^{1}$ Surgery, ${ }^{2}$ Anaesthesia, ${ }^{3}$ Pathology and ${ }^{4}$ Radiology, Col- \\ lege of Health Sciences, University of Ghana Medical School, P. O. Box GP4236, Accra, Ghana.
}

\begin{abstract}
SUMMARY
Although endocrine causes of secondary hypertension are relatively uncommon, medical practitioners must maintain a high index of suspicion for them in certain categories of patients. Such patients include young individuals, those with difficult-to-treat hypertension and those presenting with symptoms, clinical signs and/or laboratory parameters well-known to be associated with Cushing's syndrome, Conn's syndrome or phaeochromocytoma.

This paper reports on 5 patients identified over a 2-year period with various hormonally-active adrenal adenomas causing hypertension in an environment where, hitherto, the occurrence of these conditions was generally thought to be rare. Aspects of the patients' histories, examination and laboratory findings that drew attention to the possibility of the diagnosis in each case are highlighted, as are the confirmatory investigations and management methods used by a multidisciplinary team of medical practitioners. The clinical outcome with appropriate treatment of adrenalrelated hypertension is good and can result in significant cost savings in the long term.
\end{abstract}

Keywords: Secondary hypertension, adrenal adenoma, Cushing's syndrome, Conn's syndrome, primary hyperaldosteronism, phaeochromocytoma.

\section{INTRODUCTION}

The vast majority of patients with hypertension have primary or essential hypertension for which no cause can be found. In contrast, patients with secondary hypertension have an underlying disorder that is a direct cause of the hypertension. In a study from a large blood pressure clinic, secondary forms of hypertension accounted for $10.2 \%$ of all cases seen over an 18-year period. These included renovascular hypertension (3.1\%), primary hypo- thyroidism (3.0\%), a serum creatinine $>2.0 \mathrm{mg} / \mathrm{dl}$ $(1.8 \%)$, primary hyperaldosteronism $(1.4 \%)$, Cushing's syndrome $(0.5 \%)$ and phaeochromocytoma $(0.3 \%)^{1}$. In that study endocrine disorders accounted for $5.2 \%$ of all cases of hypertension and slightly more than half the cases of secondary hypertension.

To date only a few isolated cases of secondary hypertension related to hormone-secreting adrenal tumours have been published in Ghana, all of these being on phaeochromocytomas ${ }^{2,3,4}$. However, since the establishment of a referral clinic for endocrine and metabolic disorders at the Korle $\mathrm{Bu}$ Teaching Hospital (KBTH) in Accra in 1997, several patients with a variety of hormonally-active adrenal tumours have been diagnosed.

We discuss in this paper 5 patients with adrenalinduced hypertension identified between June 2001 and August 2003 who were investigated, successfully managed and followed up for a minimum of 2 years by a multidisciplinary team of medical practitioners.

\section{CASE REPORTS}

Case 1

A 13-year old female patient was referred to the Endocrine and Metabolic Clinic (EMC) of the Korle Bu Teaching Hospital (KBTH) as a case of Cushing's syndrome for evaluation. She had had appendicectomy 5 months earlier at another hospital, and although post-operative wound healing was delayed, no reason was immediately assigned to this. However, at subsequent outpatient reviews her surgeons noted that she had cushingoid facies and hypertension. Further tests requested confirmed glycosuria, hyperglycaemia and an elevated morning plasma cortisol. Her parents had also noticed excessive weight gain in the preceding year associated with easy fatigability, polyuria and re-

* Author for correspondence

fofei@africaonline.com.gh 
current boils. Her clinical presentation is summarized in Table 1.

During admission at the KBTH tests confirmed the previous finding of an elevated morning plasma cortisol (996 nmol/L-normal range 138-690) together with a persistent elevation following a standard high-dose dexamethasone suppression test (HDDST), indicating a high probability that the minal bilateral subcostal (roof-top) incision under general anaesthesia supplemented with intermittent epidural analgesia. Immediately after surgery, she was given 6-hourly intravenous injections of hydrocortisone to prevent an adrenocortical crisis. Her BP normalized without antihypertensive medications 2 days after surgery while the requirements for insulin normalised by post-operative day 10.

Table 1 Summary of patients' clinical findings

\begin{tabular}{|c|c|c|c|c|c|}
\hline $\begin{array}{c}\text { Case } \\
\text { No. } \\
\end{array}$ & $\begin{array}{c}\text { Age } \\
\text { (years)/ } \\
\text { Sex } \\
\end{array}$ & $\begin{array}{c}* \text { Diag } \\
\text { nosis }\end{array}$ & Clinical findings on presentation & $\begin{array}{c}\begin{array}{c}\text { Longest width } \\
\text { of adenoma } \\
(\mathrm{mm})\end{array} \\
\end{array}$ & $\begin{array}{c}\text { Duration of } \\
\text { postoperative } \\
\text { admission (days) }\end{array}$ \\
\hline 1 & $13 / \mathrm{F}$ & $\mathrm{CS}$ & $\begin{array}{l}\mathrm{BMI}=27.9 \mathrm{~kg} / \mathrm{m}^{2} \text {, poor wound healing, moon } \\
\text { facies, striae on arms and trunk, ecchymosis, } \\
\text { severe lower limb proximal myopathy, BP } \\
170 / 130 \mathrm{mmHg} \text {, random blood glucose } \\
22.8 \mathrm{mmol} / \mathrm{L} \text {, plasma potassium } 3.0 \mathrm{mmol} / \mathrm{L}\end{array}$ & 40 & 14 \\
\hline 2 & $57 / \mathrm{F}$ & $\mathrm{CS}$ & $\begin{array}{l}\text { BMI }=32 \mathrm{~kg} / \mathrm{m} 2 \text {, truncal obesity, severe prox- } \\
\text { imal myopathy and supraclavicular fat pads. } \\
\text { No moon facies or striae. BP } 160 / 100 \mathrm{mmHg} \text {, } \\
\text { Normal thyroid function, glycated haemoglo- } \\
\text { bin } 9.4 \% \text {, plasma potassium, } 2.6 \mathrm{mmol} / \mathrm{L} \text {. }\end{array}$ & 40 & 17 \\
\hline 3 & $29 / \mathrm{F}$ & $\mathrm{CN}$ & $\begin{array}{l}\text { Asymptomatic, BMI }=19.4 \mathrm{~kg} / \mathrm{m}^{2}, \mathrm{BP} 170 / 100 \\
\mathrm{mmHg} \text { plasma sodium }-136 \mathrm{mmol} / \mathrm{L} \text {, potas- } \\
\text { sium }-2.0 \mathrm{mmol} / \mathrm{L} \text {, urea- }-2.7 \mathrm{mmol} / \mathrm{L} \text { and creati- } \\
\text { nine } 92 \text { micromol } / \mathrm{L} \text {. Normal plasma cortisol } \\
\text { and } 24 \text {-hour urine VMA }\end{array}$ & 50 & 10 \\
\hline 4 & $46 / \mathrm{F}$ & $\mathrm{PH}$ & $\begin{array}{l}\text { Pre-operative BPs between } 100 / 80 \mathrm{mmHg} \text { and } \\
240 / 140 \mathrm{mmHg} \text {. No café au lait spots or neu- } \\
\text { rofibromata. Normal blood area, electrolytes } \\
\text { and creatinine. Pre-operative } 24 \text {-hour urine } \\
\text { VMA } 23.5 \mathrm{mg} \text { (normal range } 1.9-9.8 \text { ) }\end{array}$ & 60 & 11 \\
\hline 5 & 19/M & $\mathrm{PH}$ & $\begin{array}{l}\text { Pre-operative BPs between } 120 / 70 \text { and } \\
190 / 130 \mathrm{mmHg} \text {. No family history of hyper- } \\
\text { tension or sudden deaths, no café au lait spots } \\
\text { or neurofibromata. Pre-operative } 24 \text {-hour } \\
\text { urine VMA } 28.5 \mathrm{mg} \text { (normal range } 1.9-9.8 \text { ) }\end{array}$ & 85 & 12 \\
\hline
\end{tabular}

hypercortisolism was not pituitary-dependent ${ }^{5,6}$. Subsequent imaging of the adrenals by abdominal ultrasonography and computerized tomography (CT) scanning showed a right adrenal tumour.

Preoperatively, the elevated blood pressure (BP), hypokalaemia and hyperglycaemia were controlled with oral nifedipine, oral potassium chloride and subcutaneous injections of premixed insulin, respectively. At surgery, the right adrenal tumour was approached and removed through an abdo-
Macroscopically the adrenal mass was ovoid, smooth and orange in colour. The microscopic findings were consistent with an adrenal cortical adenoma. No mitoses or areas of necrosis were present.

Prior to discharge from hospital the IV hydrocortisone was changed to prednisolone $10 \mathrm{mg}$ orally in the morning and $5 \mathrm{mg}$ in the evening. This initial dose of prednisolone was tapered off after 3 months and finally discontinued 6 months after removal of the tumour. She remained normoten- 
sive and normoglyaemic with normal morning plasma cortisol levels up to 2 years after surgery.

\section{Case 2}

A 57 year old woman who had a personal and family history of hypertension and type 2 diabetes mellitus was under regular review at the physician specialists' outpatients' clinic at a tertiary hospital. She had had the above conditions for 20 and 4 years respectively and had also been diagnosed with combined dyslipidaemia and obesity. The referral for endocrine evaluation was prompted by her complaints of progressive weakness in the proximal muscles and slowing of body movements over a 12-month period which led her physicians to consider possibilities of Cushing's syndrome and hypothyroidism. No biochemical confirmation of these diagnoses was available at the time of the referral. From her hospital records her BP and blood glucose had been uncontrolled for several months. A week prior to the referral she was admitted to the hospital's emergency unit with uncontrolled hyperglycaemia and a left lobar pneumonia. The clinical findings are shown in Table 1.

At the EMC-KBTH the morning plasma cortisol was elevated $(866 \mathrm{nmol} / \mathrm{L})$ and remained so $(817$ $\mathrm{nmol} / \mathrm{L}$ ) after a standard low-dose dexamethasone suppression test (LDDST), confirming the presence of hypercortisolism ${ }^{5,6,7}$. CT scans of the pituitary and adrenals revealed a left adrenal mass but no pituitary lesion. The adrenal mass was also clearly demonstrated on a pre-operative abdominal ultrasound scan.

The surgical approach was through an abdominal $1 \frac{1}{2}$ roof-top incision under general anaesthesia. The intra-and post-operative periods were uneventful with introduction of parenteral hydrocortisone soon after removal of the tumour. The patient was discharged on post-operative day 17 on prednisolone $15 \mathrm{mg}$ daily in divided doses in addition to her previous medications consisting of premixed insulin, amlodipine and lisinopril.

Her insulin injections were discontinued 6 months after surgery. Blood glucose was subsequently controlled with metformin $1 \mathrm{gm}$ daily for 12 months and later on diet alone. Blood pressure at follow up ranged between 100/60 and 130/80 $\mathrm{mmHg}$ requiring a reduction in the dose of amlodipine. The dose of prednisolone was tapered off and finally discontinued 16 months after surgery.

\section{Case 3}

A 29-year old woman was referred from a private general practice clinic to the EMC-KBTH with a 5-year history of hypertension and a differential diagnosis of phaeochromocytoma and Conn's syndrome on account of considerable fluctuations noted in her BP recordings over several years. The referring doctor also noted hypokalaemia on several tests requested previously by other colleagues although the patient was not on diuretic therapy. Assessments on 24-hour urine samples for vanillylmandelic acid (VMA) had shown normal results on two occasions while both an abdominal ultrasound and CT scan had shown a right supra-renal mass.

At the EMC-KBTH an empirical diagnosis of Conn's syndrome from a right adrenal adenoma was made based on the findings of hypertension in the presence of severe hypokalaemia and an adrenal mass, normal plasma cortisol and urine catecholamine metabolite levels. Plasma renin and aldosterone levels could not be measured. The clinical findings are shown in Table 1.

The surgical approach was similar to the previous case. At operation a right adrenal tumour, that was located mainly behind the inferior vena cava, was identified and removed en-bloc. Macroscopically the tumour measured $50 \mathrm{~mm}$ across and showed features on microscopy consistent with an adrenal cortical adenoma.

The post-operative period was uneventful. She was discharged from hospital without antihypertensive medication on post-operative day 10 and finally discharged to the care of her general practitioner after 2 years of regular follow up had shown her $\mathrm{BP}$ and plasma potassium levels to have remained normal without medication.

\section{Case 4}

A 46-year old woman, diagnosed with both type 2 diabetes and hypertension a year earlier, was referred from a diabetes clinic to the EMC-KBTH with a presumptive diagnosis of phaeochromocytoma on account of a 10-month history of palpitations, headaches, excessive sweating and recurrent episodes of vomiting associated with an elevated urine VMA result. An abdominal ultrasound scan reported no abnormal findings. Her blood glucose levels were well controlled on diet only. She took nifedipine retard 20mg BD and prazosin $2 \mathrm{mg}$ BD for the hypertension (See Table 1 for the clinical presentation). 
A CT scan of the abdomen (Figure $1 \& 2$ ) and preoperative abdominal ultrasound scan both clearly demonstrated a right adrenal mass. Adrenalectomy for a diagnosis of phaeochromocytoma was carried out through a $1 \frac{1}{2}$ roof-top incision. Intra-operative BPs, which ranged between 200/130 and 60/30 $\mathrm{mmHg}$, were controlled by infusions of nitroprusside for the hypertensive episodes or ephedrine and noradrenaline for the hypotensive episodes. The BP stabilized at about $110 / 60 \mathrm{mmHg}$ without further injections of vasoactive agents after ligation of all blood vessels related to the tumour. However, on post-operative day 5 the $\mathrm{BP}$ was noted to be persistently above $140 / 90 \mathrm{mmHg}$, albeit stable, prompting the reintroduction of antihypertensive medication. She was discharged on the $12^{\text {th }}$ post-operative day. Histologically, there was no evidence of mitotic activity in the tumour.

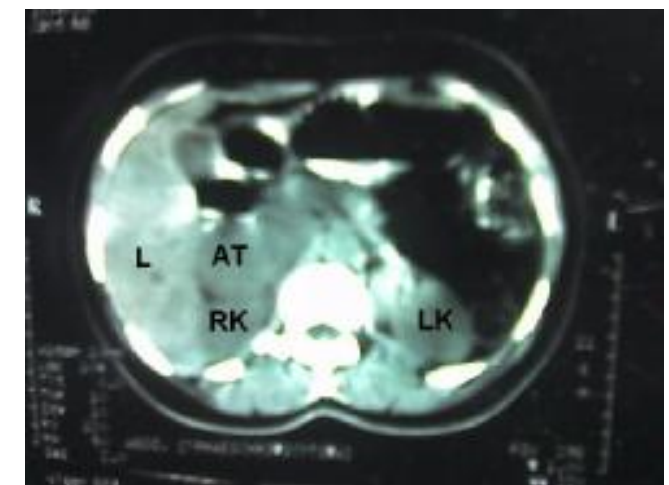

AT (Adrenal Tumour), L (Liver), LK (Left Kidney), RK (Right Kidney)

Figure 1 Abdominal CT scan (without contrast) showing right adrenal tumour in Case 4.

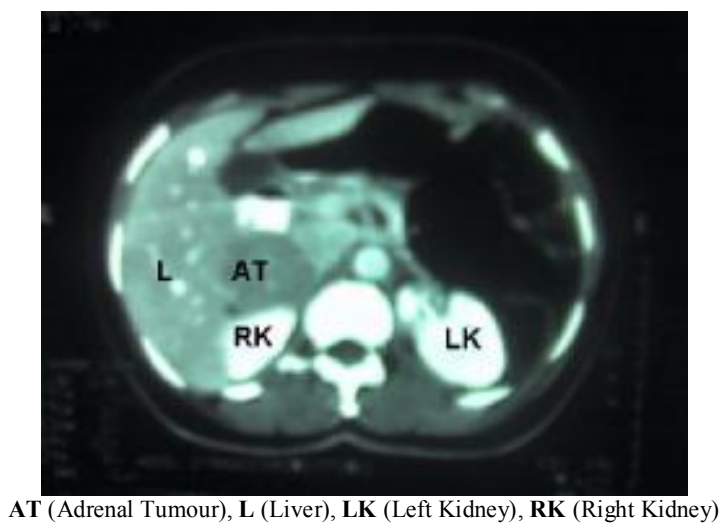

Figure 2 IV contrast-enhanced abdominal CT scan in the same patient, Case 4

At subsequent out-patient reviews BP measurements remained reasonably stable between $120 / 80$ and 140/100 on candesartan $8 \mathrm{mg}$ and bendrofluazide $2.5 \mathrm{mg}$ once daily with no recurrence of the presenting symptoms. Urine VMA 18 months postoperatively was normal at $6.3 \mathrm{mg} / 24$ hours.

\section{Case 5}

A 19-year old male student was referred from a polyclinic to the EMC-KBTH with a presumptive diagnosis of phaeochromocytoma on account of a finding of severe hypertension associated with recurrent episodes of headaches, palpitations, vomiting, excessive sweating and diarrhoea over a 1year period. He had earlier presented to a hospital in Togo where investigations had revealed a left supra-renal mass on both ultrasonography and CT imaging. No antihypertensive therapy had been initiated. A pre-operative 24-hour urine VMA showed significant elevation.

At the outpatients, he continued to have wide BP swings despite a combination of carvedilol $25 \mathrm{mg}$ twice daily and doxazosin $4 \mathrm{mg}$ once daily. He was therefore admitted for stabilization and adrenalectomy. This was carried out through a left full subcostal and a partial right subcostal incision. The findings at operation were that of a large adrenal tumour extending on to the diaphragm and making an indentation on the left kidney. Macroscopically it was an encapsulated brownish soft tissue mass which on section had a yellowish-brown colour. Microscopically, there was no tumour necrosis or mitotic activity. He was discharged 11 days postoperatively and has remained normotensive without medication to date. Urine VMA done 6 months postoperatively was $6.8 \mathrm{mg} / 24$ hours.

\section{DISCUSSION}

These 5 cases of benign, but hormonally active, adrenal tumours presented to the endocrine clinic of the KBTH over a 2-year period. Previous reports in the local literature have indicated only isolated cases of hormonally active adrenal tumours, especially pharochromocytomas ${ }^{2,3,4}$ perhaps suggesting a sporadic occurrence of adrenal tumours in Ghana and the rarity of those producing hormones other than catecholamines. While the identification of multiple and varied cases seeks to dispel this impression, this report further demonstrates the gains in suspecting the presence of adrenal tumours and in the collaboration between general practitioners and hospital specialists in identifying and managing such patients.

The clinical manifestations of adrenal tumours are variable and depend on their size, presence of malignancy or excess hormone secretion, and for that matter, which specific hormone is being overproduced. Hypertension, which was present in all our 
patients, is a common clinical denominator of hormonally-active adrenal tumours. In 3 of the patients (2 with phaeochromocytoma, 1 with Conn's syndrome), the BP elevations were episodic despite treatment compliance. One other patient was a teenager with a BP uncharacteristic for her age while another was a middle-aged diabetic woman with deterioration in BP control over time.

Other associated clinical manifestations in our patients which drew attention to the possibility of secondary hypertension needing further investigation were the recurrent headaches, palpitations, excessive sweating and vomiting in the patients with phaeochromocytoma, the persistent hypokalaemia in the woman with Conn's syndrome and the various features associated with the Cushing's phenotype noted in the two other patients.

While it will not be prudent to screen all individuals presenting with hypertension for an adrenal or hormone-related cause, a selective approach that targets patients who are young or unresponsive to standard antihypertensive treatments or exhibit features well known to be associated with Cushing's syndrome, Conn's syndrome or phaeochromocytoma for further investigations or a specialist referral would be reasonable.

For each of the three clinical scenarios presented by our patients there were multiple possibilities regarding either the location (e.g. adrenal, pituitary or ectopic) or nature (e.g. hyperplasia, adenoma or carcinoma) of the lesion, with each requiring a different management strategy. Therefore, determining not only the hormonal abnormality, but also the exact site and type of lesion was of prime importance in each case. Subsequently, as indicated by the earlier endocrine investigations, a careful study of the adrenals using appropriate imaging techniques yielded rewarding results.

Ultrasonography is an effective procedure for identifying adrenal tumours, giving results similar to $\mathrm{CT}$ and magnetic resonance imaging for lesions measuring more than $30 \mathrm{~mm}$ in diameter ${ }^{8}$. The procedure is relatively inexpensive and widely available in Ghana. Providing the ultrasonographer detailed information when requesting a test could increase the yield. In our patients a pre-operative ultrasound scan demonstrated the adrenal tumour in each case even when it had been missed in an earlier request (Case 4).

Although the CT scan images following the injection of IV contrast medium showed clearer deline- ation of the adrenal tumour in our patients (Figure 1 and 2), IV contrast material must be used

with caution when a phaeochromocytoma is suspected as the procedure may induce a hypertensive crisis. The prior administration of oral alpha- and beta-adrenoceptor antagonists can prevent this crisis. Also, the use of non-ionic contrast material is safe and does not produce significant elevation in serum catecholamine levels ${ }^{9}$.

Surgical removal is the treatment of choice for all adrenal tumours that are clinically or hormonally active $^{10}$. This intervention often provides a cure for the associated metabolic abnormalities, as well as hypertension, if present.

In long-standing adrenal-dependent Cushing's syndrome there is atrophy of the contralateral adrenal gland owing to negative feedback suppression of pituitary corticotrophin by the excess cortisol produced by the tumorous gland. Administration of parenteral corticosteroids is therefore imperative immediately after removal of the tumour, to be followed by oral formulations when the patient is ready to begin oral feeding. Gradual reduction of the oral corticosteroid replacement dose over several months will often allow recovery of normal endogenous cortisol production from the remaining non-tumorous gland.

In the case of phaeochromocytomas, residual nonparoxysmal hypertension may persist in about onethird of patients after successful surgery ${ }^{11,12}$, as occurred in our patient (Case 4), or the tumour may recur ${ }^{12}$. Patients with phaeochromocytoma should therefore have periodic clinical and biochemical follow-up indefinitely even after successful surgery ${ }^{12}$.

The surgical approach in all our patients was an open adrenalectomy through a transverse abdominal subcostal incision. Laparoscopic techniques are increasingly being used for the surgical removal of uncomplicated adrenal tumours at many centres worldwide $^{13}$. In our hospital, laparoscopic techniques for general surgical procedures have only recently been introduced and have not as yet been applied to adrenalectomy.

This report demonstrates the need for a heightened clinical suspicion and thorough investigation of patients with uncontrolled hypertension by the combined efforts of doctors in family care and specialist settings. The clinical outcome with surgical treatment of adrenal-related hypertension is 
good and can translate into significant cost savings in the long term.

\section{REFERENCES}

1. Anderson GH, Blakeman N, and Streeten DH. The effect of age on prevalence of secondary forms of hypertension in a blood pressure clinic. J Hypertens 1994; 12(5): 609-615.

2. Addy JH. Phaeochromocytoma clinically diagnosed in a Ghanaian woman. Ghana Med $J$ 1972; 11: 183-185

3. Henderson $\mathrm{K}$, Baddoo $\mathrm{H}$. The anaesthetic management of a case of phaeochromocytoma. Ghana Med J 1995; 29: 664-669.

4. Hesse A, Renner L, Onuoha C, Boatey J. Paediatric Phaeochromocytoma- A case report and literature review. Ghana Med J 2003; 37(4): 170-172.

5. Liddle GW. Tests of pituitary-adrenal suppressibility in the diagnosis of Cushing's syndrome. J Clin Endocrinol Metab 1960; 20: 1539-1561.

6. Newell-Price J, Trainer P, Besser GM, Grossman AB. The diagnosis and differential diagnosis of Cushing's syndrome and pseudoCushing's states. Endocr Rev 1998; 19: 647672.

7. Wood PJ, Barth JH, Freedman DB, Perry L, Sheridan B. Evidence for the low dose dexamethasone suppression test to screen for
Cushing's syndrome-recommendations for a protocol for biochemistry laboratories. Ann Clin Biochem 1997; 34: 222-229.

8. Suzuki Y, Sasagawa, Suzuki H, Izumi T, Kaneko H, Nakada T. The role of ultrasonography in the detection of adrenal masses: comparison with computed tomography and magnetic resonance imaging. Int Urol Nephrol 2001; 32(3): 303-306.

9. Mukherjee JJ, Peppercorn PD, Reznek RH, Patel V, Kaltsas G, Besser M, Grossman AB. Phaeochromocytoma: effect of non-ionic contrast medium in CT on circulating catecholamine levels. Radiol Jan 1997; 202(1): 227231.

10. Grumbach MM, Biller BM, Braunstein GD, Campbell KK, Carney JA, Godley PA, Harris EL, Lee JK, Oertel YC, Posner MC, Schlechte JA, Wieand HS. Management of the clinically inapparent adrenal mass ("incidentaloma") Ann Intern Med Mar 2003; 138(5): 424-429.

11. Sapienza P, Cavallaro A. Persistent hypertension after removal of adrenal tumours. Eur $J$ Surg Mar 1999; 165(3): 187-192.

12. Plouin PF, Chatellier G, Fofol I, Corvol P.. Tumour Recurrence and Hypertension Persistence after successful Phaeochromocytoma operation. Hypertension 1997; 29: 1133-1139. 